\title{
Intraoperative thrombolysis for massive pulmonary embolism during regional and general anaesthesia.
}

\author{
P. Goffin P, H. Remy, J. Guntz \\ Anaesthesia \& Critical Care Department - CHC de Liège, Belgium
}

\section{INTRODUCTION}

Acute pulmonary embolism (PE) is responsible to more than 300.000 deaths per years in Europe.

Perioperative PE presents a diagnostic challenge because signs and symptoms are insidious in a patient under general anaesthesia. Moreover, the pathophysiologic course is affected both by procedure and anaesthesia.

\section{CAse presentation}

A 63 year-old man was admitted to our hospital for an elective total hip arthroplasty. His medications include antihypertensive and oral antidiabetic tablets. He has no allergy. His past medical history is significant for a contralateral hip arthroplasty 2 years earlier without complication. There is no history of thromboembolic events.

The current procedure unfolds without complication. Eight days post surgery, the patient falls and fractures his femur along the prosthesis. Two days later, he is scheduled for open reduction and fixation. This second procedure is performed under general anaesthesia. Patient benefits from one shot femoral nerve block with ultrasound guidance.

After 120 minutes of uncomplicated procedure, the patient shows signs of mild hypotension with desaturation without any response to ephedrine and crystalloid fluid boluses. Five minutes later, he develops refractory hypotension and hypocapnia. Cardiopulmonary resuscitation (CPR) is start with a pulseless electrical activity. Despite resuscitation efforts (epinephrine, dobutamine, chest compression system with LUCAS ${ }^{8}$ ) the return of spontaneous circulation is not achieved.

A bedside echocardiography demonstrates significant signs of pulmonary embolism. 40 minutes after protocolized CPR, spontaneous circulation is not re-established. Despite the intraoperative context, we decide to perform a fibrinolytic treatment as last survival therapy. As the surgeons summarily close the surgical site, we quickly insert a central venous line. 45 minutes after onset of CPR, we inject alteplase fibrinolytic agent (Actylyse ${ }^{\circledR} ;$ Boehringer Ingelheim); $10 \mathrm{mg}$ bolus followed by $90 \mathrm{mg}$ into 60 minutes. Patient is then transferred to intensive care unit (ICU) with the chest compression system ongoing. Return of spontaneous circulation achieved within 80 minutes in the ICU ; with a total CPR of 125 minutes.
Two days later, the patient is extubated. At days 4 , he returns to the theatre to complete the osteosynthesis surgery. The patient is discharged from ICU at day 32 ; without surgical haemorrhagic complication as well as regional anaesthesia technique (no hematoma or femoral nerve complication). At one year follow-up, he is alive without neurological deficit.

\section{Discussion}

Treatment of PE involves multidisciplinary discussion in ranking priorities as a delays in diagnosis or therapy may result in fatal deterioration. European guidelines* recommend considering use of fibrinolytic therapy when cardiac arrest is caused by proven or suspected acute PE. Given the bleeding risk associated with thrombolytic therapy, the procedure should only be administrated after balancing the potentials benefits of improved outcomes and bleeding risk complications. Decision may be challenging as in this intraoperative major procedure.

Perioperative period lead to a prothrombotic state cause by inflammatory response to surgery. On the other hand, perioperative period itself is a relative contraindication for the performance of thrombolysis in case of thrombotic emergency. But fibrinolysis, as in this case, may be a last resort in life threatening cases with severe ventricular failure and shock.

We performed a 125 minutes resuscitation with successful issue. In the literature, few cases report similar situation. A high quality resuscitation gave us a chance for fibrinolysis therapy to succeed. In literature, it appears that the need for longer resuscitation is associated with worse outcome ${ }^{\star \star}$. But decision to continue or stop CPR should not be base solely on the duration of resuscitation but may be integrate in the whole context.

Concerning loco-regional anaesthesia, latest recommendations contraindicate $^{\star * \star}$ thrombolytic therapy within 48 first hours after peripherical nerve block (PNB). But in case of superficial PNB, early procedure case be evaluate with considering risk and benefit for the patient. No date are available in case of thrombolysis directly after PNB.

\section{CONCLUSION}

We performed a successful extra-long resuscitation as few describe in the literature. We used intraoperative fibrinolysis without surgical haemorrhagic complication as well as with regional anaesthesia technique.

\begin{tabular}{|c|c|c|c|c|c|c|}
\hline & $\begin{array}{c}\text { Start } \\
\text { surgery }\end{array}$ & $\begin{array}{c}\text { Early } \\
\text { deterioration }\end{array}$ & $\begin{array}{c}\text { During } \\
\text { CRP }\end{array}$ & End CPR & 3 hours after \\
\hline \multirow{5}{*}{ 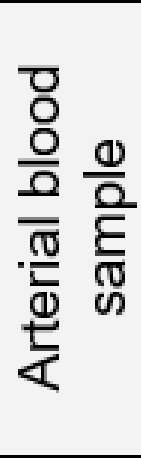 } & $\mathrm{pH}$ & 1 & 7.16 & 6.85 & 6.95 & 7.05 \\
\hline & $\mathrm{pCO}_{2}(\mathrm{mmHg})$ & 1 & 54 & 105 & 73 & 49 \\
\hline & $\mathrm{pO}_{2} \quad(\mathrm{mmHg})$ & 1 & 160 & 73 & 100 & 105 \\
\hline & $\begin{array}{l}\text { Lactate } \\
(\mathrm{mmol} / \mathrm{L})\end{array}$ & I & 1.8 & 5.1 & 13 & 11.8 \\
\hline & $\begin{array}{l}\text { Haemoglobin } \\
(g / d L)\end{array}$ & 10.3 & 8.5 & 8.2 & 7.9 & 10.3 \\
\hline \multicolumn{2}{|c|}{ End tidal CO2 } & $\overline{34}$ & 15 & $\overline{21}$ & 30 & 36 \\
\hline \multicolumn{2}{|c|}{ Entropy (SE) } & 55 & 2 & 20 & No available & No available \\
\hline
\end{tabular}

